\title{
Manejo odontológico integral de infante comprometido de forma sistémica en centro quirúrgico de Lima, Perú: reporte de caso
}

\author{
Comprehensive dental management of a child with \\ endangered health due to systemic disease and hospitalized \\ in a surgical center in Lima, Peru: Case report
}

\author{
Roxana Patricia López-Ramos ${ }^{1} \bowtie$, Gilmer Torres Ramos ${ }^{1}$, \\ Daniel José Blanco-Victorio ${ }^{1}$
}

${ }^{1}$ Facultad de Odontología, Universidad Nacional Mayor de San Marcos, Lima, Perú.

Calle Jirón Yungay 1663 Chacra Ríos Norte, Cercado de Lima, Lima, Perú. Teléfono: +51975453623. Correo electrónico: roxi_Ipz@hotmail.com

Recibido: 10 de abril del $2017 \quad$ Aprobado: 29 de mayo del $2017 \quad$ Disponible en línea: 1 de enero del 2018

Cómo citar este artículo: López Ramos RP, Torres Ramos G, Blanco Victorio DJ. Manejo odontológico integral de infante comprometido de forma sistémica en centro quirúrgico de Lima, Perú. Reporte de Caso. Rev Nac Odontol. 2018;14(27), enero 2018. doi: https://doi.org/10.16925/od.v14i27.2344

\section{Resumen}

Introducción: Ios infantes que padecen múltiples enfermedades sistémicas son más propensos a presentar problemas en la cavidad bucal al no ser diagnosticados y tratados de forma oportuna. Además, la falta de conocimiento por parte de los profesionales de salud y de los padres sobre la repercusión de estos problemas bucales puede complicar aún más el estado de estos pacientes y afectar de manera negativa la calidad de vida del menor.

Objetivo: describir el manejo odontológico integral de un infante comprometido de forma sistémica en centro quirúrgico.

Caso clínico: paciente de tres años y cinco meses de edad, de sexo femenino, con diagnóstico de cardiopatía congénita, desnutrición crónica y leishmaniasis cutánea. A nivel estomatológico presenta pulpitis irreversible y necrosis pulpar. Dada la complejidad del caso fue sometida a tratamiento odontológico integral en el centro quirúrgico del Instituto Nacional de Salud del Niño, en Lima, Perú.

Conclusión: el manejo odontológico integral, en especial en infantes que padecen enfermedades múltiples y complejas, se debe realizar de forma oportuna en un ambiente adecuado y con el apoyo de un equipo multidisciplinario de salud.

Palabras clave: diente, cardiopatía, desnutrición, infante, leishmaniasis, tratamiento. 


\title{
Comprehensive dental management of a child with endangered health due to systemic disease and hospitalized in a surgical center in Lima, Peru: Case report
}

\begin{abstract}
Introduction: Children who suffer multiple systemic diseases are more likely to have problems in their oral cavity because of not being diagnosed and treated in a timely manner. In addition, health professionals and parents' lack of knowledge about the repercussions of these oral problems can trigger even more burdens in these patients' health conditions and negatively affect the children's quality of life.

Objective: To describe the comprehensive dental management of a child with endangered health due to systemic disease and hospitalized in a surgical center.

Clinical case study: A female patient aged three years and five months, diagnosed with congenital heart disease, chronic malnutrition and cutaneous leishmaniasis. In stomatological terms, she presents irreversible pulpitis and pulpal necrosis. Given the complexity of the case, she underwent comprehensive dental treatment at the National Institute of Child Health surgical center, in Lima, Peru.

Conclusion: Comprehensive dental management, especially in children suffering from multiple and complex diseases, must be carried out in a timely manner, in an appropriate environment and with the support of a multidisciplinary health team.
\end{abstract}

Keywords: tooth, heart disease, malnutrition, child, leishmaniasis, treatment.

\section{Manejo odontológico abrangente de uma criança com doenças sistêmicas em um centro cirúrgico em Lima, Peru: relato de caso}

\section{Resumo}

Introdução: as crianças que sofrem múltiplas doenças sistêmicas são mais propensas a ter problemas na cavidade bucal por não serem diagnosticadas e tratadas oportunamente. Além disso, a falta de conhecimento por parte dos profissionais de saúde e dos pais sobre a repercussão desses problemas bucais pode complicar ainda mais a condição desses pacientes e afetar negativamente a qualidade de vida da criança.

Objetivo: descrever o manejo odontológico abrangente de uma criança com doenças sistêmicas em um centro cirúrgico.

Caso clínico: paciente de três anos e cinco meses, sexo feminino, com diagnóstico de cardiopatia congênita, desnutrição crônica e leishmaniose cutânea. Ao nível estomatológico, apresenta pulpite irreversível e necrose pulpar. Dada a complexidade do caso, ela passou por tratamento odontológico abrangente no centro cirúrgico do Instituto Nacional de Salud del Niño, em Lima, Peru.

Conclusão: 0 manejo odontológico abrangente, especialmente em crianças que sofrem de doenças múltiplas e complexas, deve ser realizado oportunamente, em um ambiente adequado e com o apoio de uma equipe multidisciplinar de saúde.

Palavras-chave: dente, cardiopatia, desnutrição, criança, leishmaniose, tratamento. 


\section{Introducción}

Las enfermedades sistémicas presentan manifestaciones a nivel bucal, en las cuales su repercusión puede empeorar el cuadro clínico. Por tanto, el odontólogo, el médico, el paciente y la familia deben trabajar de forma conjunta, a fin de procurar una mejor calidad de vida de los pacientes infantes, ya que ellos se encuentran en desarrollo y crecimiento. La práctica cotidiana de la odontología contemporánea debe incluir el conocimiento y la identificación de las enfermedades sistémicas que se presentan en los pacientes [1], con el fin de realizar un correcto manejo odontológico, y en un ambiente adecuado cuando se trata de pacientes complejos. Además, concientizar sobre salud bucal en relación con la higiene bucal correcta y los buenos hábitos alimenticios, entre otros, tanto a los padres de familia como a su entorno. Es por esto que el objetivo de este reporte de caso es describir el manejo odontológico integral de un infante comprometido de forma sistémica en centro quirúrgico.

Entre las enfermedades sistémicas que se pueden presentar se encuentran las alteraciones cardíacas. Para estas, a nivel mundial se estima una prevalencia de 0,5 a 9 por 1000 nacidos vivos; en Latinoamérica nacen cada año 54000 niños con cardiopatías congénitas [2], y en el Perú la incidencia es de ocho a diez casos por cada 1000 niños nacidos vivos [3]. Entre las alteraciones tenemos la atresia tricúspidea e hipoplasia ventricular derecha, las cuales se caracterizan por una malformación cardiaca congénita y por presentar un cuadro anatomo-clínico más o menos característico: falta de desarrollo del ventrículo derecho con atresia o hipoplasia de la válvula tricúspide y de la arteria pulmonar, de forma conjunta con una comunicación interauricular. Así, entonces, presenta un mayor trabajo del ventrículo izquierdo y su hipertrofia demostrable a la radiología y a la electrocardiografía [4]. Otra alteración cardiaca es la persistencia del conducto arterial (PCA), la cual se refiere a la persistencia de la permeabilidad del conducto arterioso posterior a la sexta semana de vida intrauterina. En un alto porcentaje (40\%) la PCA se encuentra asociada con otras cardiopatías, principalmente con las comunicaciones interventricular e interauricular, la válvula aórtica bivalva, la estenosis pulmonar (valvular y supravalvular) y la coartación de aorta [5].
También se encuentran las alteraciones nutricionales. Se presenta desnutrición, la cual se da, principalmente, por deficiencia calórica y proteica. Según la Unicef, en el mundo existen 146 millones de niños menores de cinco años con desnutrición infantil [6]; en Perú, la prevalencia de la desnutrición crónica es del 14,4\% [7]. Repercute de manera desfavorable en el desarrollo de niños y niñas durante su primera infancia, dado que afecta el sistema inmunológico, de manera que disminuye la resistencia a casi todas las enfermedades y aumenta el riesgo de morbilidad y mortalidad infantil [8]. Unicef la define como una emergencia silenciosa: genera efectos muy dañinos que se manifiestan a lo largo de la vida de la persona, y no se detectan de inmediato. La primera señal es el bajo peso, seguido por la baja altura; sin embargo, son solo las manifestaciones más superficiales del problema.

Existen también problemas dermatológicos, uno de los cuales es la leishmaniasis, lo cual constituye un gran problema de salud pública puesto que es endémica en 88 países del mundo, aproximadamente, con una incidencia anual de 1 a 1,5 millones de casos al año [9]; en el Perú la tasa de incidencia anual (TIA) es de 8,2 x 100000 habitantes [10]. La leishmaniasis es una zoonosis causada por parásitos del género Leishmania. Presenta varios patrones clínicos: leishmaniasis cutánea, cutánea difusa, mucocutánea y visceral [9].

Las manifestaciones bucales encontradas en pacientes con enfermedades sistémicas son diversas, y en las cardiopatías congénitas son: cianosis de mucosas bucales (se presenta, sobre todo, en encías, mucosa alveolar y paladar, y se debe a la deficiencia de oxígeno en la sangre, por lo que esta toma una coloración azulada) [11]; retraso en la erupción dental, el cual es proporcional al retraso en el desarrollo físico del paciente; hipoplasias del esmalte (son poco comunes, pero se reportan en la literatura); y caries dental, como una manifestación secundaria que puede deberse a la falta de cuidado con la salud bucal por factores inherentes al paciente. Asimismo, afección multisistémica, sobreprotección de los padres, presencia de factores sociales como, por ejemplo, pertenecer a familia disfuncional, o condiciones socioeconómicas desfavorables.

En la desnutrición infantil puede ser un factor de riesgo que afecta el desarrollo del aparato estomatognático, lo que incluye los dientes y sus estructuras de sostén. De igual forma, puede incidir tanto de manera directa como indirecta en la presencia 
de caries en edad temprana, debido a que una dieta con déficit de proteínas, vitaminas y minerales debilita las estructuras dentales y puede ocasionar alteraciones en la producción de saliva. También puede producir anomalías relacionadas con la forma, el tamaño y la estructura dentaria, y repercutir, además, en la dentición permanente [12].

En cuanto a la leishmaniasis, todas las formas clínicas pueden comenzar con lesiones primarias en la región cervical —algunas veces afectan la cavidad bucal一, o ciertos síntomas significativos detectados por el odontólogo. Tales síntomas incluyen dificultades en la deglución, disfonía y disnea. Por tanto, los odontólogos juegan un papel importante en el diagnóstico temprano de la leishmaniasis oral, a fin de evitar la propagación sistémica de la enfermedad [13].

Los profesionales de la salud deben promover el concepto de integralidad con los padres de familia a fin de explicar las repercusiones que un foco infeccioso en cavidad bucal puede tener en un niño no solo cardiópata, sino también sistémico. No se debe ver la condición sistémica como una patología aislada, debido a que, en sentido estricto [14], existen repercusiones que se interrelacionan, de manera que realizar siempre las interconsultas con las especialidades pertinentes contribuye al buen diagnóstico, al tratamiento y al pronóstico, en el propósito de mejorar la calidad de vida del paciente $\mathrm{y}$ de su entorno familiar.

\section{Reporte de caso}

Paciente femenino de tres años y cinco meses de edad, de sexo femenino, procedente de una provincia ubicada en la Sierra del Perú. Acude a la Unidad de Pacientes Medicamente Comprometidos (PMC) del Servicio de Odontoestomatología del Instituto Nacional de Salud del Niño, Lima-Perú, por interconsulta del servicio de cardiología para eliminación de focos infecciosos; presenta como diagnóstico principal cardiopatía congénita, desnutrición crónica y leishmaniasis cutánea. La madrerefiere que su hija será operada del corazón y presenta varios dientes cariados que requieren tratamiento.

En los antecedentes prenatales presentó peso al nacer de 3000 gr, parto eutócico con controles prenatales incompletos; en antecedentes natales presentó lactancia materna artificial diurna y nocturna hasta los dos años de edad, aproximadamente; y en antecedentes patológicos leishmaniasis cutánea (enero del 2016), hipoplasia tricúspidea, persistencia del conducto arterial (PCA), y desnutrición crónica (octubre del 2016).

En el examen físico general presentó: saturación de oxigeno del 74-80\%; frecuencia cardiaca de 108 por min; frecuencia respiratoria de $36 \times \mathrm{min}$; peso de 11,600 kg; piel tibia, húmeda, elástica, cianosis en labios y mucosas, acrocianosis, cinco lesiones ulcero costrosas profundas con cicatriz atrófica en la hemicara del lado derecho (siete meses aproximadamente de evolución, en los que recibió como tratamiento anfotericina $\mathrm{B}$ ); en torax y pulmones murmullo vesicular, pasa bien en auscultación cardiopulmonar, no se presentan ruidos agregados; capacidad vital de buena intensidad, soplo sistólico II/VI; abdomen blando depresible; ruidos hidroaéreos presentes; con relación al sistema nervioso central se encuentra despierta, relacionada con el entorno.

En el examen clínico estomatológico extrabucal se evidenció una paciente mesofacial, con simetría facial y con crecimiento normodivergente. Se observan lesiones ulcerosas cicatrizantes profundas a nivel de la hemicara derecha (figura $1 \mathrm{~A}$ ). Al examen intrabucal presentó una dentición decidua, gingivitis moderada, mucosas bucales cianóticas, con múltiples lesiones cariosas activas en esmalte y dentina en piezas anteriores y posteriores de ambas arcadas, y con lesiones periapicales en las piezas 51, 52, 61, 62, 75 y 84 . Higiene bucal mala (figura 1в). Paciente con conducta definitivamente negativa, según la escala de Frankl; rechaza el tratamiento, grita con fuerza y es temerosa.

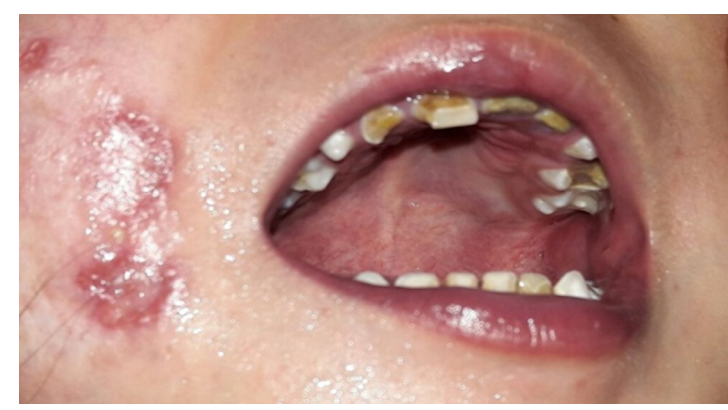

Figura 1. A. Se observa hemicara derecha con lesiones eritematosas. Labios delgados, cianóticos y resecos. Mucosa yugal cianótica, vascularizada, móvil. Paladar duro: ovalado, profundidad intermedia y cianótica Fuente: elaboración propia 


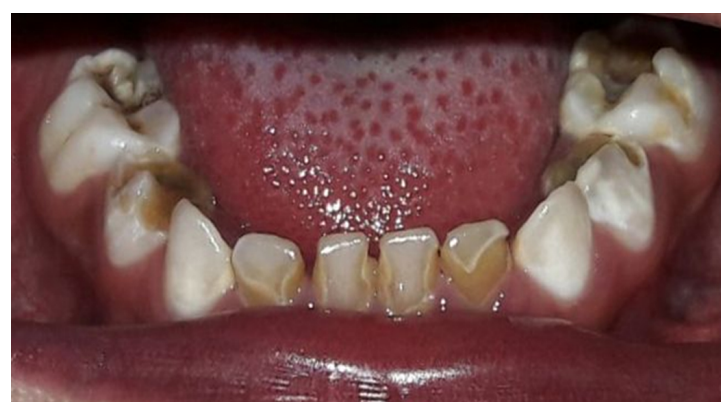

Figura 1. B. Se observa lengua saburral, móvil, humedecida. Dientes: con lesiones cariosas múltiples y destrucción coronaria amplia con evidente compromiso pulpar

Fuente: elaboración propia

Se solicitaron exámenes auxiliares de laboratorio, ecocardiografía a solicitud de cardiología y radiografía panorámica (véanse las tablas 1 y 2 , y la figura 2).

Tabla1. Exámenes de laboratorio solicitados previa intervención quirúrgica

\begin{tabular}{lc}
\hline \multicolumn{1}{c}{ Exámenes de laboratorio } & $\begin{array}{c}\text { Valor del } \\
\text { paciente }\end{array}$ \\
\hline Hematocrito & $68 \%$ \\
Leucocitos & $6,380 / \mathrm{mm}^{3}$ \\
Segmentados & $40 \%$ \\
Linfocitos & $68 \%$ \\
Plaquetas & $150000 / \mathrm{mm}^{3}$ \\
Hemoglobina & $18 \mathrm{~g} / \mathrm{dl}$ \\
Glucosa & 80 \\
Creatinina & $41 \mathrm{mg} / \mathrm{dl}$ \\
Tiempo de protrombina & $15,5 ”$ \\
Tiempo parcial de tromboplastina activada & $42,3 ”$ \\
Segmentados & $20 \%$ \\
\hline
\end{tabular}

Tabla 2. Ecocardiografía solicitada previa intervención quirúrgica

Interpretación

Atresia pulmonar, hipoplasia tricúspidea, PCA, sinusiodes coronarios.

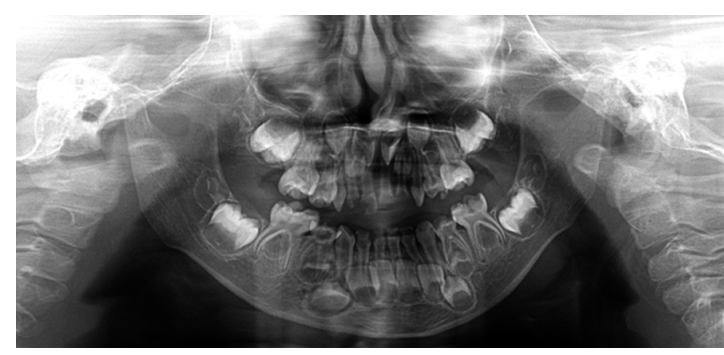

Figura 2. Radiografía panorámica. Se observan estructuras óseas sin alteraciones

Fuente: elaboración propia

Dentro del protocolo para el tratamiento odontológico integral en el centro quirúrgico se solicitaron interconsultas para evaluación y sugerencias correspondientes a pediatría, cardiología y anestesiología, las cuales indicaron riesgo quirúrgico grado III (enfermedad sistémica grave con limitación funcional definida, pero que no constituye una amenaza constante para la vida). Además, cardiología solicitó premedicación como profilaxis antibiótica una hora antes de la intervención y dos horas posintervención (cefalotina de $1 \mathrm{~g}$ vía endovenosa) $[14,15]$. También se solicitó el consentimiento al responsable del cuidado del menor para la intervención quirúrgica en centro quirúrgico bajo anestesia general.

El tratamiento odontológico integral consistió en:

- Fisioterapia antes de la intervención, se instruyó a la madre sobre hábitos de higiene oral.

- Adecuación del medio bucal con gasa más clorhexidina al 0,12\%.

- Restauraciones con resina compuesta en piezas dentarias: 55, 54, 64, 65, 74, 75, 71, 72, 81, 82, 84 y 85 .

- Coronas de celuloide en piezas dentarias: 51, 52, $61,62,71,72,81,82$.

- Tratamientos pulpares:

- Pulpotomías en piezas dentarias: 55, 54, 64, $65,74,75,84,85$.

- Pulpectomías en piezas dentarias: 51, 52, 61, 62 (véase la figura 3). 


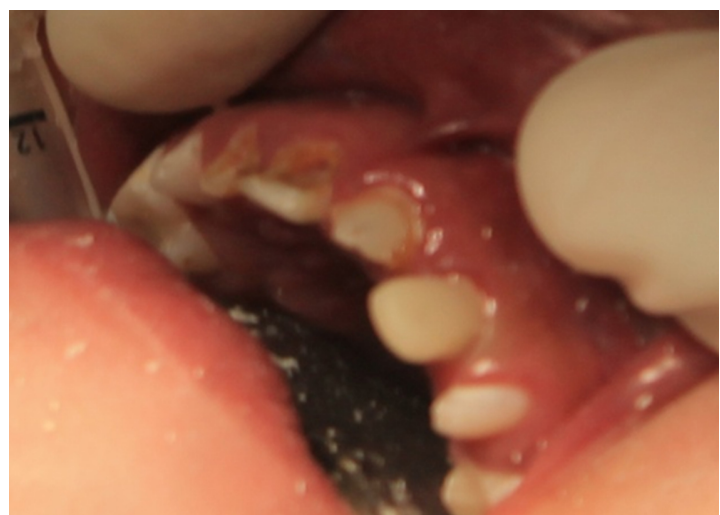

Figura 3a. Se procedió a realizar la remoción de tejido carioso; luegose realizó la apertura cameral, la irrigación, el secado y la preparación biomecánica de los conductos de las piezas del sector antero superior

Fuente: elaboración propia

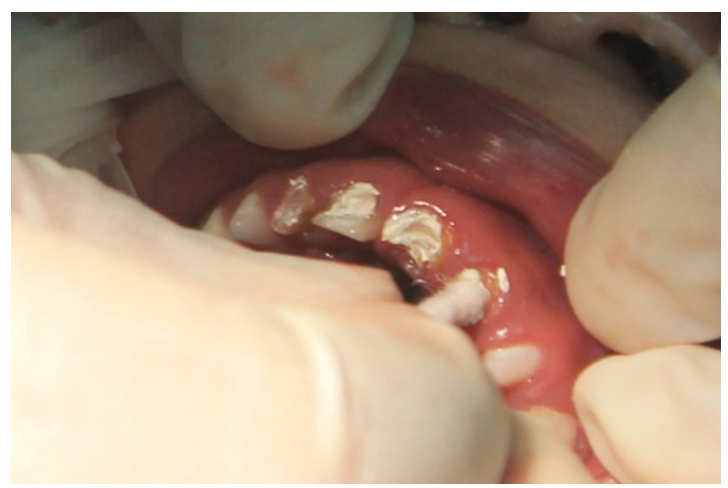

Figura 3b. Colocación de material obturador en conductos de piezas antero superiores

Fuente: elaboración propia

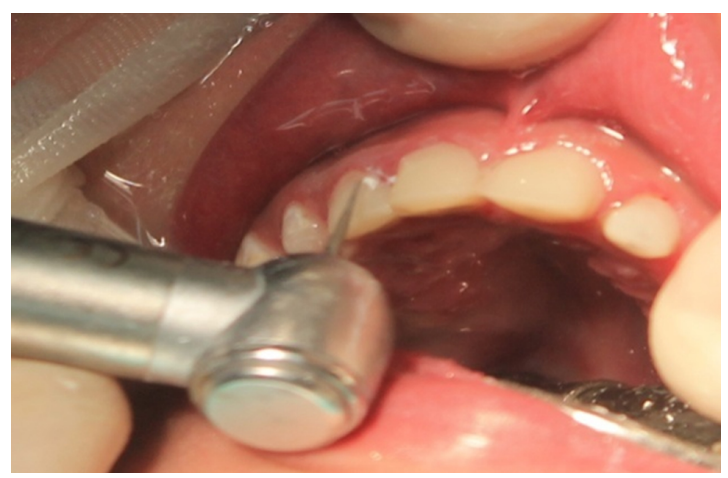

Figura 3c. Colocación de coronas celuloides en los conductos depiezas del sector antero superior

Fuente: elaboración propia
Se realizó el control postratamiento odontológico integral, en cuya evaluación se apreció mejoría moderada en la higiene oral; se instruyó a la madre a mejorar a fin de evitar posibles recidivas. La paciente se encontró asintomática, recuperando la función masticatoria, fonética y estética (figura 4). Se prescribieron aplicaciones de flúor barniz cada tres meses, controles radiográficos dentro de tres meses y, luego, cada seis meses.

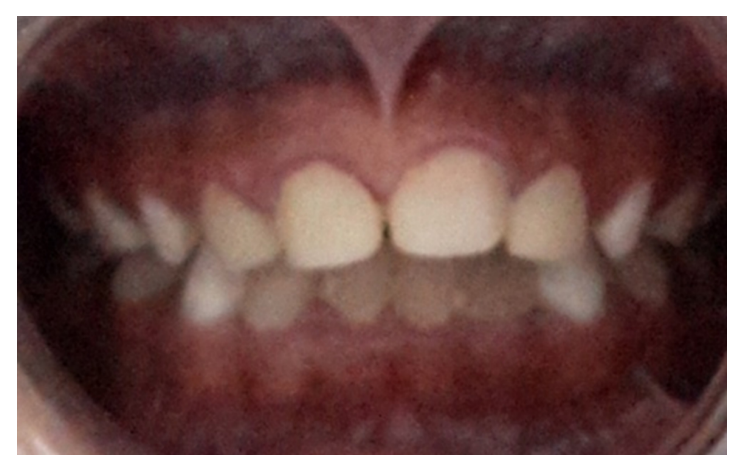

Figura 4. Control postratamiento odontológico integral. Fuente: elaboración propia

\section{Discusión}

Los pacientes con enfermedades sistémicas son susceptibles de presentar mayores problemas bucales, cuyo tratamiento es más complejo. Por esto, es preferible realizarlos en un centro quirúrgico bajo anestesia general, puesto que los tratamientos ambulatorios demandan mayor tiempo operatorio y la cooperación del paciente infante es en un mayor grado negativa, según la escala de Frankl [16] (la más empleada en odontología). Por esta razón, al planificar el tratamiento odontológico integral se debe evaluar la complejidad del estado tanto sistémico como bucal. En este reporte de caso se optó por su realización en un centro quirúrgico bajo anestesia general, debido a las múltiples enfermedades sistémicas y la no cooperación del paciente a los procedimientos odontológicos ambulatorios; se contó con las medidas preventivas adecuadas e interconsultas con otras especialidades médicas en busca del éxito del tratamiento, cuyos resultados se observaron al cabo de un mes, pues no solo la salud bucal del paciente mejoró de forma notable, sino también su conducta se tornó positiva. 
Por otra parte, las cardiopatías congénitas existen de diferentes tipos, cada una con sus características y su sintomatología propias. No obstante, todas representan una condición que debe identificar el odontólogo, previo al tratamiento, y deben tomarse las precauciones pertinentes con respecto al manejo. Entre las más importantes se resalta la necesidad de profilaxis antimicrobiana, con el uso de medicamentos tales como amoxicilina por vía oral con dosis de $50 \mathrm{mg} / \mathrm{kg}$, y ampicilina, cefazolina o ceftriaxona vía intramuscular o endovenosa con dosis de $50 \mathrm{mg} / \mathrm{kg}$; cuando no se tolera por vía oral y en alérgicos a las penicilinas, clindamicina en dosis de $20 \mathrm{mg} / \mathrm{kg}$, azitromicina o claritromicina en dosis de $15 \mathrm{mg} / \mathrm{kg}$ por vía oral; y el uso de oxígeno suplementario en casos de cardiopatías congénitas cianóticas $[14,15,17]$. En el reporte se utilizó cefalotina como profilaxis antimicrobiana y se tomó como precaución, en caso de una hemorragia, el empleo de transfusión de las plaquetas. Toda condición gingivo-periodontal inflamatoria debe ser controlada antes de programar cualquier procedimiento de tipo electivo. Los antisépticos deben utilizarse para uso cotidiano, entre tanto, antisépticos más potentes, como, por ejemplo, clorhexidina al $0,12 \%$, deben emplearse una vez a la semana. Los conteos bacterianos se reducen de forma significativa con un enjuague de clorhexidinaal $0,12 \%$ durante dos minutos (o de yodopovidona), antes de iniciar cualquier procedimiento.

Estudios realizados reportaron que los niños con malnutrición presentaron mayor incidencia de patologías bucales, tales como gingivitis y enfermedad periodontal; coincidieron en que a mayor severidad de la desnutrición, se pueden presentar mayores problemas dentales como, por ejemplo, caries y gingivitis $[18,19]$. Se observaron en el presente reporte hallazgos similares a lo mencionado. Otros estudios hallaron que los niños con problemas de malnutrición por defecto a edad temprana presentaron alteraciones del esmalte desde el nacimiento, como, por ejemplo, dens in dente, agenesias, taurodontismo, dientes en forma cónica, supernumerarios, microdoncias, transposiciones, fusiones, mesiodens, dientes retenidos e hipoplasia del esmalte $[19,20]$.

Zavala et al. mencionan que los niños afectados por algún padecimiento sistémico son pacientes en quienes su atención odontológica se descuida de manera importante, ya que toda la atención se enfoca en su padecimiento sistémico y la salud bucal pasa a segundo término. Esto sucede, en general, en razón al desconocimiento por parte de los familiares y del personal médico de la relación estrecha que existe entre la salud bucal y los padecimientos sistémicos, lo que da como resultado pacientes con múltiples patologías bucales que, en ciertas situaciones, repercuten de forma negativa en el manejo y el control de su enfermedad sistémica [11]. Asimismo, la falta de conocimiento de buenos hábitos de higiene bucal puede exacerbar el cuadro clínico del paciente sistémico.

En el presente reporte se relacionan tratamientos pulpares de acuerdo con la guía clínica de la Unidad de Pacientes Medicamente Comprometidos-Servicio Odontoestomatología del Instituto Nacional de Salud del Niño. La realización de tratamiento pulpar es controversial; la American Academy of Pediatric Dentistry (AAPD) no ofrece recomendación para la terapia pulpar en pacientes con cardiopatías [21]. Según Johnson et al., el manejo de los dientes primarios o permanentes con compromiso pulpar en pacientes cardiacos requiere la evaluación del tipo de afección cardíaca y el riesgo de bacteremia del procedimiento dental planificado. Los autores afirman que la endocarditis infecciosa rara vez está relacionada de forma directa con los procedimientos dentales, y que la incidencia y la magnitud de la bacteriemia, cuando la instrumentación del canal no se extiende hacia los tejidos periapicales, es baja; la mayoría de las bacterias se eliminan de la sangre en 10 minutos [22]. El tratamiento alternativo para los dientes primarios con compromiso pulpar es la extracción y la colocación posible de un mantenedor de espacio [23]. Sin embargo, se recomienda la precaución con respecto a la colocación de zapata distal como mantenedor de espacio en pacientes con anomalías cardiacas que requieran profilaxis antibiótica antes del tratamiento dental, debido a la posibilidad de inflamación crónica asociada o patología periodontal. Sin embargo, no se puede comparar el grado de bacteriemia que ocurre durante la extracción y la posible fuente crónica de acumulación bacteriana alrededor de un mantenedor de espacio en comparación con la terapia pulpar [23]. No se han publicado ensayos aleatorios y controlados con animales, y tampoco ensayos en humanos sobre la incidencia de bacteriemia después de una terapia pulpar en comparación con una extracción y el posterior desarrollo de endocarditis en asociación con cardiopatías. Los resultados del estudio de 
Dunlop et al. sugieren la necesidad de desarrollar una guía formal de la AAPD para el manejo y el tratamiento dental en pacientes pediátricos con enfermedad cardíaca [21].

Es fundamental la educación y concientizar a los familiares sobre la conservación de la salud bucal, con igual énfasis en que los controles sean periódicos. Por esto, es labor del odontólogo fomentar la atención en salud oral en todos los pacientes comprometidos de forma sistémica, como parte fundamental de su tratamiento integral $[11,24,25]$.

\section{Conclusiones}

El conocimiento de las distintas enfermedades sistémicas y sus manifestaciones clínicas a nivel bucal brindará al odontólogo la capacidad de planificar y ejecutar el tratamiento de manera adecuada. En este propósito es importante apoyarse en el equipo multidisciplinario en salud, a fin de que remitan temprano a los niños que tienen compromiso sistémico, y así, con educación en salud oral, el odontólogo y su equipo brinde educación oportuna y seguimiento periódico a estos pacientes, y concientice a la familia y al entorno sobre la necesidad de prevenir y mantener una adecuada salud oral en pacientes sistémicos.

\section{Referencias}

[1] Secretaría de Salud de Bogotá. Guía de práctica clínica en salud oral infancia y adolescencia. Colombia; 2010. Disponible en: [http://www.saludcapital.gov.co/DSP/Documentos\%20Salud\%20Oral/ Gu\%C3\%ADa\%20de\%20Pr\%C3\%A1ctica\%20 Cl\%C3\%ADnica\%20en\%20Salud\%20Oral\%20Infancia-Adolescencia.pdf]

[2] Sandoval N. Cardiopatías congénitas en Colombia y en el mundo. Rev Colomb Cardiol. 2015;22(1):1-2. doi: 10.1016/j.rccar.2015.03.004

[3] Olortegui A, Adrianzen M. Incidencia estimada de las cardiopatías congénitas en niños menores de 1 año en el Perú. An Fac Med. 2007 abrjun;68(2):113-124.
[4] Duffau G. Atresia tricúspidea e hipoplasia ventricular derecha. Rev Chil Pediatr. 1950;21(6):266-278.

[5] San Luis et al. Guía de práctica clínica Persistencia del conducto arterioso. Rev Med Inst Mex Seguro Soc. 2012;50(4):453-63.

[6] Fondo de Naciones Unidas para la Infancia-Unicef. Progreso para la infancia: un abalance sobre la nutrición; 2006

[7] Instituto Nacional de Estadística e Informática-INEI. Desnutrición crónica infantil en niñas y niños menores de cinco años disminuyó en 3.1 puntos porcentuales. Lima; 2016; 49: 3.

[8] Beltrán A, Seinfeld J. Desnutrición crónica Infantil en el Perú: un problema persistente. Documento de discusión. Lima: Universidad del Pacífico; 2009. p. 54.

[9] Martínez E,Tejada D, Rivas E, Mansilla J, Miller $\mathrm{K}$, Pérez $\mathrm{O}$, et al. Leishmaniasis cutánea diseminada: a propósito de un caso. Dermatología. CMQ. 2011;9(2):107-110.

[10] C Yon. Situación epidemiológica de la leishmaniasis en el Perú. SE. 2017;26(45):1454-5.

[11] Zavala KB, Vinitzky I, Ramírez D. Manejo estomatológico del paciente pediátrico con cardiopatía congénita. Revisión de la literatura. Univ Odontol. 2011 ene-jun;30(64):57-66. Disponible en: http:// www.redalyc.org/html/2312/231218581007/

[12] Torres L, Duque J, Granada J, Serna M, García R. Anomalías dentales y su relación con la malnutrición en la primera infancia: un análisis crítico de literatura. Rev Nac Odontol. 2015;11(20):65-69. doi: http://dx.doi.org/10.16925/od.v11i20.941

[13] Mignogna MD, Celentano A, Leuci S, Cascone M, Adamo D, Ruoppo D, et al. Mucosal leishmaniasis with primary oral involvement: a case series and a review of the literature. Oral Dis. 2015 en;21(1):708. doi: https://doi.org/10.1111/odi.12268

[14] Rodríguez MS, Durán A, De la Teja E, Palacios A, Osnaya H. Rehabilitación bucal bajo anestesia generalen pacientes cardiópatas. Experiencia de cuatro años en el Instituto Nacional de Pediatría. Acta Pediatr Mex. 2012; 33(2):71-75.

[15] Wilson W et al. Prevention of infective endocarditis. Guidelines from the american heart association: A guideline from the american heart association rheumatic fever, endocarditis, and kawasaki disease committee. Research Interdisciplinary Working Group. Circulation. 2007;116:1736-54. doi: https://doi.org/ 10.14219/jada.archive.2008.0346 
[16] Lopera Ossa MP, Cardeño Chaverra V, Muñetones Correa D, Serna Salazar E, Díaz Garavito MV, Bermúdez Reyes P. Manejo de la conducta del paciente pediátrico por estudiantes de la Facultad de Odontología, Universidad Cooperativa de Colombia, Medellín, 2012. Rev Nac Odontol.2013;9(16):59-65.

[17] Sivertsen TB, Aßmus J, Greve G, Åstrøm AN, Skeie MS. Oral health among children with congenital heart defects in Western Norway. Eur Arch Paediatr Dent. 2016 oct;17(5):397-406. doi: https://doi. org/10.1007/s40368-016-0243-y

[18] Espinal G. Estudio retrospectivo de anomalías dentales y alteraciones óseas de maxilares en niños de cinco a catorce años de las clínicas de la Facultad de Odontología de la Universidad de Antioquia. Rev Fac Odontol Univ Antioq. 2009;21(1):50-64. doi: http://hdl.handle.net/10495/4978

[19] Souza MA, Soares Junior LAV, Santos MA, Vaisbich MH. Dental abnormalities and oral health in patients with hypophosphatemic rickets. Clinics (São Paulo). 2010;65(10):1023-1026. doi: http://dx.doi. org/10.1590/S1807-59322010001000017

[20] Barrionuevo L. Anomalías dentomaxilares y factores asociados en niños con parálisis cerebral. Rev Chil Pediatr. 2008;79(3):272-80. doi: http://dx.doi. org/10.4067/S0370-41062008000300005
[21] Dunlop R, Sanders B, Jones J, Walker L, Caldwell R. Pulp therapy in pediatric patients with congenital heart disease: survey of american academy of pediatric dentistry members. J Dent Child. 2013;80(3):139-144.

[22] Johnson BR, Fischer DJ, Epstein JB. The medically complex endodontic patient. En: Ingle JI, Bakland LK, Baumgartner J, eds. Ingle's Endodontics. $6^{\text {th }}$ ed. Ontario, BC, Canada: BC Decker, Inc; 2008.

[23] Brill WA. The distal shoe space maintainer: Chair side fabrication and clinical performance. Pediatr Dent. 2002;24:561-5.

[24] Carvajal M, Lara L. Dossier manejo del paciente comprometido sistémicamente en Odontopediatría. Univ Odontol. 2011 ene-jun;30(64):17-18.

[25] Orellana Centeno JE, Delgado De Alba FA, Nava Calvillo JF, Nava Zárate N, Orellana Centeno M, Onofre Quilantán MG. Educación y cuidado estomatológico de pacientes infantiles con insuficiencia renal en pacientes de Soledad de Graciano Sánchez, San Luis Potosí. Rev Nac de Odontl. 2012;8(15): 57-62. 